\title{
ANÁLISIS DE LA PERCEPCIÓN DE LOS VISITANTES DE LA CIUDAD DE QUITO EN RELACIÓN AL ALOJAMIENTO TEMÁ́tICO BASADO EN LA MÚSICA ECUATORIANA
}

\section{ANALYSIS OF THE PERCEPTION FROM THE VISITORS OF QUITO CITY IN RELATION TO A THEMATIC LODGING BASED ON THE} ECUADORIAN MUSIC

\author{
Shirley C. Segura-Ronquillo*¹,Denisse A. Santos-Toaza', Yanina E. Patiño-Cabezas'. \\ 1 Facultad de Especialidades Empresariales, Universidad Católica de Santiago de Guayaquil, Guayaquil - Ecuador. E-mail: shirley.segura@cu.ucsg.edu.ec, \\ denissita_santos@hotmail.com, evelynpc94@gmail.com
}

El propósito del estudio fue analizar la percepción de los turistas de la ciudad de Quito en relación a un servicio de alojamiento cuyo valor agregado en la ambientación y diseño está basado en la temática de la música ecuatoriana para conocer si una propuesta de hostal temático podría ser viable en la ciudad. Se realizó una investigación descriptiva de corte cuantitativo, basado en el método deductivo con la cual se determinaron los gustos y preferencias del turista nacional y extranjero al momento de elegir su lugar de hospedaje y su grado de aceptación hacia la iniciativa de un hostal temático. Como principal resultado, el 93\% de los visitantes del destino elegirían este tipo de alojamiento alternativo para complementar su experiencia turística.

PALABRAS CLAVE: alojamiento temático, cultura musical, hostal, música ecuatoriana, turismo.
This study was developed with the purpose of analyzing the tourists' perception of Quito's city regarding to a lodging service whose added value in the setting and design is based on the theme of Ecuadorian music in order to know if a Thematic hostel proposal could be viable in the city. A quantitative descriptive research was carried out, based on the deductive method with which the tastes and preferences of national and foreign tourists were determined when choosing their place of lodging and their degree of acceptance towards the initiative of a thematic hostel. As a main result, $93 \%$ of visitors to the destination would choose this type of alternative accommodation to complement their tourist experience.

KEY WORDS: Thematic hospitality, Musical culture, Hostel, Ecuadorian music, Tourism. 


\section{INTRODUCCIÓN}

El cantón Quito de la provincia de Pichincha tiene entre sus atractivos más representativos el Centro localizado al sur de la ciudad, es uno de los sitios más concurridos de Quito y el país, con un total de visitas al 2016 de 77.851 turistas, es decir, un 60\% de aceptación Histórico (Empresa Pública Metropolitana de Gestión de Destino Turístico, 2017). Los datos obtenidos de Quito en Cifras muestran que el perfil de los turistas que más visitan la ciudad de Quito tienen edades entre 31 y 45 años que comprenden el 43\% y entre los 17 y los 31 años de edad representan el 34\%. El 61\% de los turistas que visitan Quito poseen un grado de educación de tercer nivel seguido por el 21\% que solo registró hasta el nivel de educación secundaria y el $17 \%$ ha alcanzado el nivel de estudios de postgrado.

En cuanto a la motivación, la institución gestora del turismo en la metrópolis, sugiere que el motivo principal de la visita del turista es de recreación y ocio, y corresponden a un $49 \%$ de los visitantes, seguido muy de cerca por la visita a familiares y amigos con un 31\%. También se define que la principal actividad realizada en la ciudad de Quito es la visita a lugares históricos, la cual es realizada por el $32 \%$ de los turistas.

Así, ligado al turismo cultural se analiza la teoría sobre la creatividad cuyo eje se basa en la valoración comunitaria o cultural (Gardner, 1998). Para las Naciones Unidas (2010) la creatividad es tanto un atributo propio del ser humano como un proceso para generar ideas donde sobresalen elementos como la creatividad artística y la originalidad para solucionar problemas.

En el discurso de Piedras (2006) se favorece también el ámbito cultural al afirmar que Latinoamérica posee grandes recursos como la multiculturalidad y las tradiciones latentes en las diferentes actividades socioeconómicas que se desarrollan, un entorno de convivencia armónica, tolerancia y mutuo reconocimiento constituyendo un insumo invaluable que puede articular diversas estrategias de desarrollo regional y local (citado por Santos \& Patiño, 2016).

Es así como, la cultura es un eje transversal en el desarrollo de actividades de infraestructura, sociales y humanas tanto en el ámbito urbano como rural por lo cual es necesario el apoyo al desarrollo de las estrategias nacionales culturales (Banco Mundial, 2008). De esta manera, el turismo es un medio para aprovechar los elementos culturales, y a través del alojamiento, crear propuestas de valor para el turista y generar un servicio que diversifique su oferta y aporte al desarrollo económico de la ciudad (Santos \& Patiño, 2016).

Dentro de la terminología de interés para reconocer la aplicación del concepto de hospedaje temático, se revisaron varias acepciones entre ellas, de La Real Academia Española (RAE) en la cual define la palabra temático como algo "que se arregla, ejecuta o dispone según el tema o asunto de cualquier materia”. Teniendo en cuenta esta definición, la investigación como tal buscó combinar el aspecto hotelero anteriormente mencionado con la experiencia de la música, constituyéndose así un concepto de servicio de hostal temático de la música nacional.

Por otro lado, dentro del ámbito de alojamiento, Plumed (2013) declara "que la hospitalidad no la iniciamos nosotros, la motivan tanto los sucesos externos como las personas que nos rodean. Nuestra tarea es saber responder" (p. 28o). De este hecho se parte para crear e innovar en productos y servicios turísticos; así lo definen Rivas y Madagán (2012) en su libro Estructura, economía y política turística para quienes "la producción turística se concentra esencialmente en la generación de servicios" (p. 58). Estos hechos permiten enfatizar nuevamente la experiencia de la música como un servicio que complementa la experiencia turística desde el alojamiento.

Adicionalmente, se puede traer a relevancia la experiencia cultural de la música como complemento dentro de la experiencia turística integral. MacCannell (2003) en su escrito El turista: una nueva 
teoría de la clase ociosa define a las experiencias turísticas como experiencias culturales, y que "el ocio se construye a partir de experiencias culturales" (Citado por Torres, 2008, p. 99). Es decir, que el valor de la experiencia proviene finalmente de los turistas y su cultura de origen así como la cultura presente en los lugares donde se efectúa la visita turística.

Para Plog (1974) los tipos de turistas pueden ser: (a) psicocéntricos que son turistas extremadamente conformistas, el turismo para ellos es un hábito adquirido, buscan zonas muy desarrolladas y no admiten cambios; (b) alocéntricos que son personas motivadas por estímulos como la aventura y buscan destinos pocos conocidos; y (c) los medicéntricos que comparten características de los dos anteriores.

La tipología de los turistas también se basa en la experiencia turística (Cohen, 1979), que divide al turista en cinco modos: (a) El modo de vagabundo para turistas altamente aventureros y viven dentro de la comunidad local; (b) El modo de recreación en el que el turista explora dentro del turismo lo que puede encontrar en otras actividades recreativas; (c) El modo de diversión donde el turista no se aleja de su centro pues el turismo no tiene significado para él y; (d) El modo de experiencia en el cual el turista se engancha con la vida auténtica de otros.

Sobre la base de los fundamentos analizados, el presente trabajo tiene el propósito de analizar la percepción de los turistas en relación a un servicio de alojamiento cuyo valor agregado esté basado en la temática de la música ecuatoriana y se reconozca como una opción turística viable.

\section{MATERIALES Y MÉTODOS}

El estudio desarrollado corresponde a una investigación de enfoque cuantitativo, de tipo descriptiva, porque se emplea el estudio de los datos obtenidos a través de los instrumentos aplicados para dar respuesta al objetivo principal y pregunta de la investigación, empleando la medición numérica y el uso de la estadística para establecer con exactitud los patrones de cierta población, mostrar las realidades y su característica fundamental (Gómez, 2006; Rodríguez, 2005).

Para el desarrollo sistemático de esta investigación se aplicó el método deductivo cuya rigurosidad permite analizar teorías de validez para determinar posibles soluciones a hechos concretos (Bernal, 2006).

Se aplicó este método porque la investigación se basa en el estudio del turismo y servicios que permitió perfilar los tipos de turistas para finalmente mediante la aplicación de los instrumentos de investigación conocer y analizar su percepción sobre los alojamientos temáticos en la ciudad de Quito.

El levantamiento de la información se realizó mediante una encuesta basada en once preguntas tomadas del estudio realizado por Santos y Patiño (2016), tanto en versión español como en inglés, para encuestar a 383 visitantes dividido entre 180 turistas nacionales y 203 turistas extranjeros que visitaron la ciudad de Quito durante las dos primeros fines de semana del mes de Julio del año 2016 y que corresponde a la temporada alta del año, que de acuerdo con los datos obtenidos de la web de Quito-turismo, éste es el periodo de mayor afluencia turística en la ciudad.

Se aplicaron los procedimientos de muestreo aleatorio e intencional, seleccionando a los turistas de manera aleatoria en el centro histórico de Quito, a quienes se les preguntó si eran visitantes o residentes de la ciudad y el idioma de su preferencia para responder a la encuesta (versión de la encuesta tanto en español como en inglés).

RESULTADOS

Para el soporte del estudio desarrollado se aplicó un cuestionario a una muestra de 383 turistas encuestados en el Centro Histórico de la ciudad de Quito, basado en once preguntas que fueron formuladas de acuerdo al análisis del entorno para conocer, entre otros aspectos, el porcentaje de aceptación del alojamiento temático por parte de los turistas nacionales y extranjeros, y los servicios que les gustaría disfrutar en un hostal temático de la música 
ecuatoriana, además, de conocer el medio y precios por el cual eligieron el hospedaje en la ciudad de Quito.

Los rangos de edad fueron establecidos de 17 a 30, de 31 a 45, de 46 a 60 y de más de 60 años, tanto para turistas nacionales como extranjeros. El 66,5\% de visitantes nacionales correspondieron a un rango de edad entre 17 y 30 años, mayoritario como el $84,5 \%$ de los visitantes extranjeros. Coincide que en promedio el $75,5 \%$ de los turistas tanto nacionales como extranjeros del centro histórico de Quito están entre los 17 y 30 años edad, la cual fue el mercado objeto de este estudio, y sólo el $10,35 \%$ están entre los 31 a 60 años y el resto son mayores a 60 años. Por otro lado, el género femenino representa el 6o,9\% de los visitantes nacionales y el $61.4 \%$ en los turistas extranjeros. Ambos resultados en promedio representan el $61,2 \%$ mujeres y el 38,8\% restante a los hombres, en- tre nacionales y extranjeros.

En relación al tipo de alojamiento preferido para la estadía, los visitantes nacionales prefieren los hostales con un $43,8 \%$ y los visitantes extranjeros con un $45,9 \%$ prefieren su hospedaje en un hotel, siendo las dos alternativas favoritas de alojamiento que en general los hostales abarcan el 44,35\% de la preferencia y los hoteles un 42,35\%.

Al momento de viajar, los visitantes nacionales determinaron que lo que más influye en su decisión de un lugar de estadía es la recomendación personal con un $35,2 \%$, en cambio, el 40,1\% de visitantes extranjeros eligió el lugar para hospedarse por la proximidad a un lugar determinado. Sin embargo, entre ambos visitantes, el $35,7 \%$ toman decisiones de hospedaje por la cercanía a un lugar determinado mientras que el 34,05\% por recomendación personal (ver Tabla 1).

Figura 1: Caracterización de turistas nacionales y extranjeros

\begin{tabular}{lccc}
\hline Variable & Nacionales & Extranjeros & Consolidado \\
\hline Edad & & & \\
\hline 17 a 30 años & $66,50 \%$ & $84,50 \%$ & $75,50 \%$ \\
31 a 45 años & $4,40 \%$ & $10,10 \%$ & $7,25 \%$ \\
46 a 60 años & $2,80 \%$ & $3,40 \%$ & $3,10 \%$ \\
+ de 60 años & $26,30 \%$ & $2,00 \%$ & $14,15 \%$ \\
\hline Género & & & \\
\hline Femenino & $60,90 \%$ & $61,40 \%$ & $31,15 \%$ \\
Masculino & $39,10 \%$ & $38,60 \%$ & $38,85 \%$ \\
\hline Hospedaje & & & \\
\hline Hotel & $38,80 \%$ & $45,00 \%$ & $93,90 \%$ \\
Hostal & $43,80 \%$ & $44,00 \%$ & $9,30 \%$ \\
Pensión & $9,60 \%$ & $9,00 \%$ & $4,90 \%$ \\
Otros & $7,80 \%$ & $2,00 \%$ & $34,05 \%$ \\
\hline Factores de decisión para la estadía & & & $35,70 \%$ \\
\hline Recomendación personal & $35,20 \%$ & $27,15 \%$ \\
Cercanía al lugar & $31,30 \%$ & $32,90 \%$ & $3,10 \%$ \\
Servicio al cliente & $33,00 \%$ & $40,10 \%$ & \\
Otro & $0,50 \%$ & $5,70 \%$ & \\
\hline
\end{tabular}

En la Tabla 2 se presenta el resumen de resultados de cuatro variables analizadas con dos opciones de respuestas.

Respecto a la pregunta cinco, durante su visita a Quito el $86,1 \%$ de los turistas nacionales y apenas el $43,5 \%$ de los extranjeros sí escucharon la música nacional pero mencionaron que no se distinguían cuál es el genero nacional contundiendolo con otros géneros de América Latina. De manera general, tanto turistas nacionales como extranjeros en promedio, el $65 \%$ han escuchado música nacional durante su paso por la ciudad de Quito.

La pregunta seis de la Tabla 2 muestra la preferencia de un hostal temático de la 
música ecuatoriana, a lo cual el 93,9\% de los turistas nacionales y el $92,8 \%$ de los visitantes extranjeros opinaron que sí le gustaría conocer la música nacional de Ecuador por medio de un hostal temático de la música. En general, el 93,35\% de los visitantes del Distrito Metropolitano de Quito están de acuerdo con conocer la música ecuatoriana a través de un hostal temático.

La séptima pregunta relacionada con los ser

vicios de valor añadidos a la propuesta temática, se presentó la idea del servicio adicional de estudio de grabación en el hostal que permitiera grabar una canción durante la estadía, a lo cual el 68,9\% de turistas nacionales y el $85,5 \%$ de los visitantes extranjeros opinaron que les gustaría contar con el servicio adicional de estudio de grabación. En promedio, al $77,2 \%$ de los visitantes del centro histórico de la capital les gustaría contar con el servicio de estudio de grabación en el establecimiento temático.

La octava pregunta mostrada en la Tabla 2 se refiere al porcentaje de visitantes que pagarían por el servicio de alojamiento en un hostal temático de la música nacional con desayuno incluido, y el $82,8 \%$ de visitantes nacionales y el $88,9 \%$ de visitantes extranjeros opinaron que si estarían dispuestos a pagar por este servicio, con un total promedio del $85,85 \%$ de todos los visitantes nacionales y extranjeros pagarían por este tipo de hospedaje con desayuno incluido.

\begin{tabular}{|c|c|c|c|}
\hline Opciones de respuesta & Nacionales & Extranjeros & Consolidado \\
\hline \multicolumn{4}{|c|}{ Pregunta 5: ¿Ha escuchado música nacional durante su visita? } \\
\hline Sí & $86,10 \%$ & $43,50 \%$ & $64,80 \%$ \\
\hline No & $13,90 \%$ & $56.50 \%$ & $35,20 \%$ \\
\hline \multicolumn{4}{|c|}{$\begin{array}{l}\text { Pregunta 6: ¿Le gustaría conocer la música nacional por medio de un hostal temático de la músic } \\
\text { nacional? }\end{array}$} \\
\hline $\begin{array}{l}\text { Sí } \\
\text { No }\end{array}$ & $\begin{array}{c}93,90 \% \\
6,10 \% \\
\end{array}$ & $\begin{array}{l}92,80 \% \\
7,20 \%\end{array}$ & $\begin{array}{l}93,35 \% \\
6,65 \% \\
\end{array}$ \\
\hline \multicolumn{4}{|c|}{ Pregunta 7: ¿Le gustaría contar con un espacio para grabar canciones que desee cantar? } \\
\hline Sí & $68,90 \%$ & $85,50 \%$ & $77,20 \%$ \\
\hline No & $31,10 \%$ & $14,50 \%$ & $22,80 \%$ \\
\hline \multicolumn{4}{|c|}{$\begin{array}{l}\text { Pregunta 8: ¿Usted pagaría por hospedarse en un hostal temático de la música nacional, co } \\
\text { desayuno incluido? }\end{array}$} \\
\hline Sí & $82,80 \%$ & $88,90 \%$ & $85,85 \%$ \\
\hline No & $17,20 \%$ & $11,10 \%$ & $14,15 \%$ \\
\hline
\end{tabular}

Por otro lado, se procedió a preguntar el rango de precios que los turistas estarían dispuestos a pagar (ver Tabla 3) y el 66,7\% de los turistas nacionales y el 66,7\% de los turistas extranjeros eligieron un rango de precios de $\$ 63$ a $\$ 79$, es decir, en promedio un $66,7 \%$ de los encuestados nacionales y extranjeros eligió el menor rango de precios, lo cual es coherente con la elección mayoritaria de hostales.

Tabla 3: Valor dispuesto a pagar por una noche de alojamiento temático

\begin{tabular}{cccc}
\hline Rango de precios & Nacionales & Extranjeros & Consolidado \\
\hline$\$ 63-\$ 79$ & $66,70 \%$ & $66,70 \%$ & $66,70 \%$ \\
$\$ 80-\$ 96$ & $27,70 \%$ & $27,50 \%$ & $27,60 \%$ \\
$\$ 97-\$ 120$ & $5,60 \%$ & $5,80 \%$ & $5,70 \%$ \\
Total & $100,00 \%$ & $100,00 \%$ & $100,00 \%$ \\
\hline
\end{tabular}


Para complementar el estudio se elaboraron las dos últimas preguntas referentes a los servicios adicionales con los que les gustaría contar dentro del hostal y los medios publicitarios que influyeron en su decisión de alojamiento.

En relación a la décima pregunta, el internet es el medio que ejerce mayor influencia en las decisiones que toman los visitantes sobre el tipo de hospedaje ele- gido (Gráfico 1), con una representación del $76,05 \%$ de todos los visitantes encuestados, destacando que los visitantes extranjeros utilizan mayoritariamente este medio con un $84,5 \%$ de encuestados que basaron sus decisiones en la información brindada por este medio. Además de los medios expuestos, también hubo un factor importante que es la recomendación personal recibida.

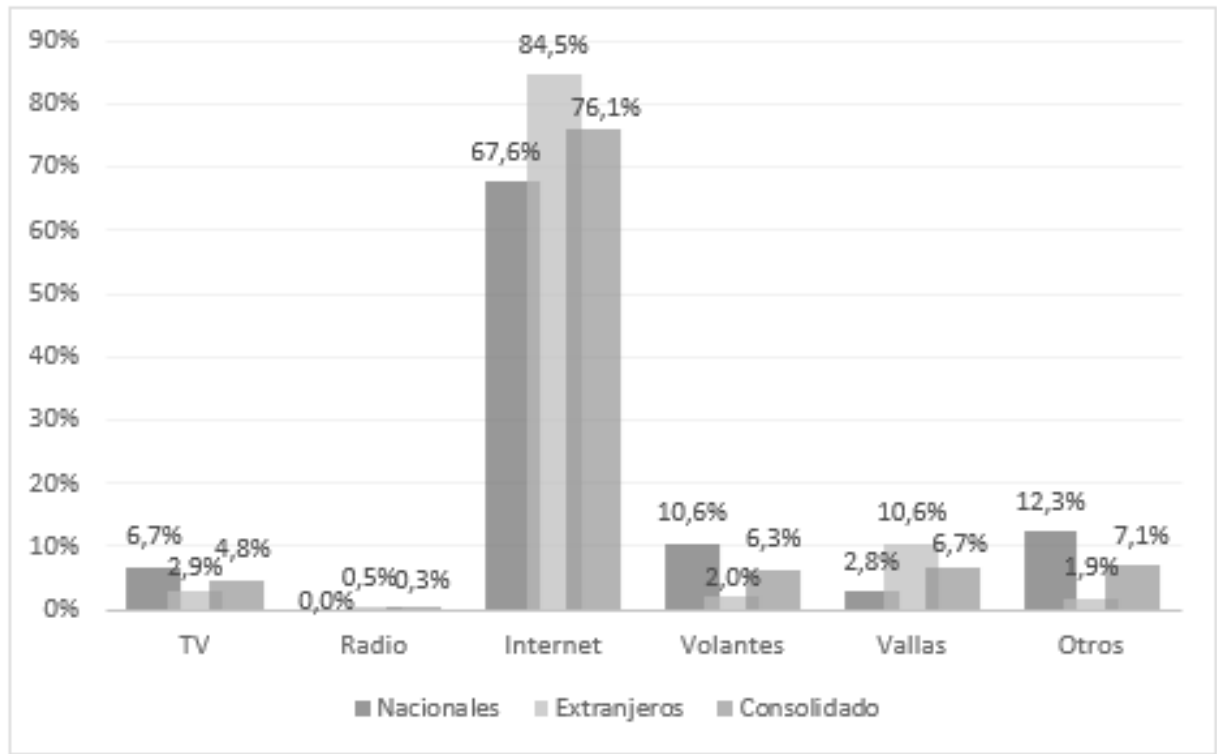

Gráfico 1: Medios publicitarios que influyeron en la decisión de alojamiento

Finalmente, se evaluaron los servicios adicionales sugeridos por los visitantes para contar con una estadía de su preferencia (Gráfico 2), entre los cuales se destacan los café concerts con un $75,4 \%$ en promedio de todos los visitantes, el uso de instrumentos musicales con un consolidado de $55,7 \%$ y el servicio de reservación para eventos culturales con un 52,6\% de aceptación.

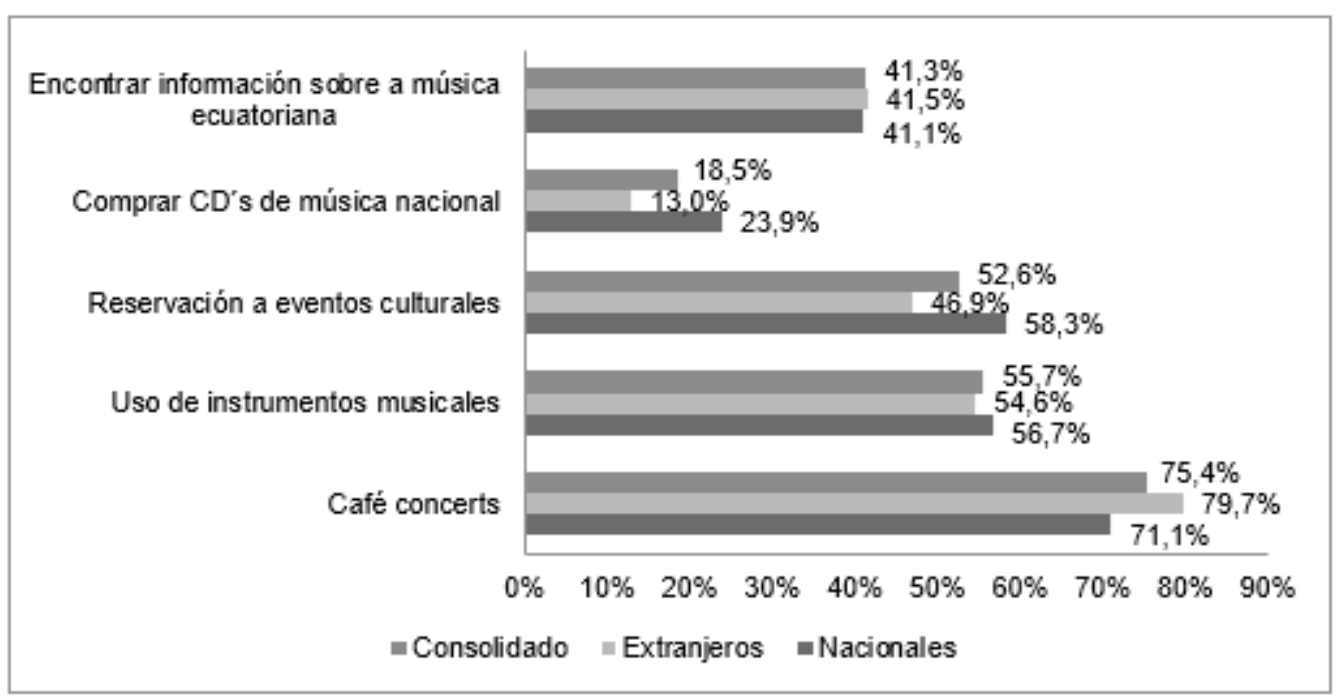

Gráfico 2. Pregunta 11: Servicios adicionales preferidos 


\section{CONCLUSIONES}

El estudio permitió conocer que la mayor parte de los turistas nacionales y extranjeros pertenecen al género femenino y están en un rango de edad entre los 17 y 30 años. Además, los visitantes han preferido hospedarse en hostales. En relación a los motivos por los cuales eligen los hostales u hoteles para la estancia en sus viajes, difiere entre nacionales y extranjeros. Para los turistas nacionales es más importante y valorada la recomendación personal y para los turistas internacionales es la proximidad a los atractivos.

En cuanto a la aceptación del alojamiento un hostal temático de la música ecuatoriana se muestra que la mayoría de visitantes les gustaría gozar de esta experiencia; en especial los visitantes del extranjero, de los cuales más de la mitad de la muestra tomada indicaron que no han escuchado nunca la música ecuatoriana durante su visita al país, concordando que todos pagarían una tarifa entre los $\$ 63$ y $\$ 79$ por una noche incluido el desayuno y servicios en un hostal temático de música nacional del Ecuador.

Finalmente se concluye que la aceptación por parte de los turistas nacionales e internacionales que visitan el Distrito Metropolitano de Quito es favorable para desarrollar cualquier emprendimiento turístico enfocado en el hospedaje temático para hostales de segunda y tercera categoría en la ciudad de Quito, lo cual presenta una oportunidad para nuevos nichos de mercado segmentados por el enfoque cultural de la música, que conecte al visitante con una de las manifestaciones culturales más destacadas del país, y a la vez poco conocidas, como es la música ecuatoriana.

\section{REFERENCIAS}

Asociación de Municipales Ecuatorianas AME (2016). Cantón Quito. Obtenido de: http://www.ame.gob.ec/ ame/index.php/ley-de-transparencia/68-mapa-cantones-del-ecuador/ mapa-pichincha/295-canton-quito.

Banco Mundial (2008). La cultura y las prioridades del banco mundial. Cultura y desarrollo: un compromiso para la libertad y el bienestar. Recuperado de: https://books.google.com.ec.

Bernal, C. (Ed). (2006). Metodología de la investigación para administración, economía, humanidades y ciencias sociales. Naucalpan: Pearson Educación.

Cohen (1979). Teoría de la experiencia del turista. La creación y promoción de experiencias en un destino turístico. Recuperado de: file:///C:/Users/Pati\%C $3 \%$ B1o/Downloads/221511-786221-1-PB.pdf.

Empresa Pública Metropolitana de Gestión de Destino Turístico (2017). Quito en cifras. Recuperado de: http:// www.quito-turismo.gob.ec/estadisticas/datos-turisticos-principales/category/82-quito-en-cifras.

Gardner (1998). Teoría de la Creatividad. Creatividad aplicada: Cómo estimular y desarrollar la creatividad a nivel personal, grupal y empresarial. Recuperado de: https://books.google.com

Gómez (2006). Enfoque cuantitativo, cualitativo y mixto. Recuperado de: http:// www.eumed.net/tesis-doctorales/2012/ mirm/cualitativo_cuantitativo_mixto. html.

MacCannell, D. (2003). El turista: una nueva teoría de la clase ociosa. Barcelona: Editorial Melusina. Obtenido de: http://www.redalyc.org/articulo. oa?id $=90215158008$.

Naciones Unidas (2010). Informe sobre la economía creativa. Contexto y contornos de la economía creativa. Recuperado de: http://www.unesco.org/culture/ $\mathrm{pdf} /$ creative-economy-report-2013-es. pdf

Piedras (2006). Diversidad cultural conceptos estrategias. Recuperado de: http:// cultura.gencat.cat/web/.content/sscc/ gt/arxius_gt/diversidad_cultural_conceptos_estrategias.pdf.

Plog (1974). Teoría de los perfiles de turistas. A propósito del turismo: La construcción social del espacio turístico. Recuperado de: https://books.google. com.ec.

Plumed, C. (2013). Archivo Hospitalario: Revista de Historia de la Orden Hospitalaria de San Juan de Dios. Una aportación para la humanización. Recuperado de: https://books. google.com.ec/books?id=KopqBAAAQBAJ\&pg $=$ PA $280 \& d q=$ teoria +de + la + hospitalidad\&hl $=e s-419 \& s a=X \&-$ ved =oahUKEw j 9 -fis 697 TA h- 
V B N i Y K H e 5 O D 5 U Q 6 A E IJ z A $\mathrm{B} \# \mathrm{~V}=$ onepage $\& \mathrm{q}=$ teoria $\% 20$ de $\% 20 l a \% 20$ hospitalidad\&f=false.

Real Academia Española (s.f.). Diccionario de la lengua española. Obtenido de: http://dle.rae.es/srv/fetch?id=ZOnOAZE

Rivas, J. \& Madagán, M. (2012). Estructura, Economía y Política Turística. España: SEPTEM ediciones.

Rodríguez (2005). Investigación descriptiva. Metodología de la Investigación. Recuperado de: https://books.google. com.ec/books.

Santos, D. \& Patiño, E. (2016). Propuesta de factibilidad de un hostal temático de la música en el sector del Centro Histórico en la ciudad de Quito. Tesis de grado. Obtenido de: http://repositorio. ucsg.edu.ec:808o/handle/3317/6441

Torres, R. (2008). Reseña de "El turista: una nueva teoría de la clase ociosa" de Dean MacCannell. Sociedad Hoy, (14), 99101. Recuperado de http://www.redalyc. org/articulo. oa?id=902151580o 8 